DOI: https://doi.org/10.46296/yc.v5i9edespdic.0139

\title{
ESTRATEGIAS DIDÁCTICAS QUE INCLUYEN LAS TICS PARA EL DESARROLLO DEL PENSAMIENTO CRÍTICO
}

\section{DIDACTIC STRATEGIES THAT INCLUDE ICTS FOR THE DEVELOPMENT OF CRITICAL THINKING}

\author{
Zambrano-Sánchez María Narcisa ${ }^{1}$; Gallego-Macías Marcos Ramón ${ }^{2}$ \\ ${ }^{1}$ Universidad San Gregorio de Portoviejo, USGP. Portoviejo, Ecuador. Correo: \\ e.mnzambrano2@sangregorio.edu.ec. ORCID ID: https://orcid.org/0000-0002-6070-9340. \\ 2 Universidad San Gregorio de Portoviejo, USGP. Portoviejo, Ecuador. Correo: \\ mgallegos@sangregorio.edu.ec. ORCID ID: https://orcid.org/0000-0002-3651-034X.
}

\begin{abstract}
Resumen
En el presente trabajo se analizó la influencia de las estrategias didácticas con la incorporación de las TICs en el desarrollo del pensamiento crítico; donde el escenario principal son los estudiantes, quienes adquieren una enseñanza centrada en la capacidad de análisis, reflexión e interpretación y en la resolución de problemas. Se planteó como objetivo determinar la incidencia de las estrategias didácticas en el desarrollo del pensamiento crítico de los estudiantes de básica superior. El diseño metodológico tuvo un enfoque cuantitativo, exploratorio y descriptivo. Se aplicaron encuestas a docentes, estudiantes y una entrevista a un profesional con experticia en pedagogía. En cuanto a los docentes se comprobó que su uso y aplicación es limitado y desactualizado, aplicando técnicas tradicionales que no dan resultados esperados. En lo que concierne a los discentes existe la falta de interés y motivación en el proceso de enseñanzaaprendizaje, obteniendo una alta demanda en estrategias innovadoras tanto para la labor docente como para el aprendizaje significativo de los estudiantes. Con la implementación de estrategias como el aprendizaje basado en proyectos, aprendizaje basado en problemas, aprendizaje colaborativo, flipped classroom, aprendizaje autónomo, entre otros. que incluyen las TICs, se fomenta el pensamiento crítico de los educandos, concibiendo en ellos la motivación, reflexión y la participación activa que les permita alcanzar un aprendizaje óptimo. El propósito del artículo es señalar las estrategias más importantes que originen competencias innovadoras y tecnológicas para lograr un cambio en la educación actual.
\end{abstract}

Palabras claves: estrategias didácticas; participación activa; pensamiento crítico; Tecnología de la información y comunicación.

\begin{abstract}
In the present work, the influence of didactic strategies with the incorporation of ICTs in the development of critical thinking was analyzed; where the main stage is the students, who acquire a teaching focused on the capacity for analysis, reflection and interpretation and on problem solving. The objective was to determine the incidence of didactic strategies in the development of critical thinking in upper secondary students. The methodological design had a quantitative, exploratory and descriptive approach. Surveys were applied to teachers, students and an interview to a professional with expertise in pedagogy. As for teachers, it was found that its use and application is limited and out of date, applying traditional techniques that do not give expected results. As far as students are concerned, there is a lack of interest and motivation in the teachinglearning process, obtaining a high demand for innovative strategies both for the teaching work and for the meaningful learning of the students. With the implementation of strategies such as project-based learning, problem-based learning, collaborative learning, flipped classroom, autonomous learning, among others. that include ICTs, the critical thinking of students is encouraged, conceiving in them the motivation, reflection and active participation that allows them
\end{abstract}

Información del manuscrito:

Fecha de recepción: 29 de octubre de 2021.

Fecha de aceptación: 08 de diciembre de 2021.

Fecha de publicación: 17 de diciembre de 2021. 
to achieve optimal learning. The purpose of the article is to point out the most important strategies that originate innovative and technological competencies for achieve a change in education today.

Keywords: didactic strategies; active participation; critical thinking; Information and communication technology.

\section{Introducción}

Este estudio abordó las estrategias didácticas y su incidencia en el desarrollo del pensamiento crítico de los estudiantes de Octavo año de EGB de la Unidad Educativa Fiscal Dr. Francisco Campos Coello del cantón Guayaquil. Estas estrategias de enseñanza incluyen las TICS para el desarrollo del pensamiento reflexivo, consideradas un paradigma en la educación actual.

El contexto de un mundo globalizado exige pensar que la educación requiere identificar las implicaciones que conllevan el uso de las estrategias didácticas, las cuales han incidido en el desarrollo del pensamiento crítico y reflexivo de los estudiantes, lo cual nos hace reflexionar sobre los beneficios que se podrán obtener en la educación actual, y así formar futuros profesionales íntegros y aptos para la sociedad. Desde la década del 70, comienzan a realizarse estudios más sistemáticos, con la elaboración y validación de inventarios de estrategias y tácticas, así como relaciones entre ellas (Monereo, 1999, pág. 5). Desde ese momento la Pedagogía intenta asumir la temática e introduce el concepto "estilos de aprendizaje", mostrando la temática con mayor importancia para los docentes.

Para la autora (Roque, 2016, pág. 10) las estrategias didácticas son procesos secuenciales ordenados y sistematizados de diferentes actividades planeadas por el docente, determinando su modo de proceder sin apartarse del objetivo propuesto y de la forma de aprender del escolar. Estas se diferencian de otras acciones y obedecen al instante en que se descubre el proceso de enseñanza - aprendizaje de la clase.

En el Ecuador el proceso de enseñanza-aprendizaje, no es el más favorable, existen estudiantes que carecen de habilidades de 
pensamiento crítico, es decir poseen limitada capacidad de tomar decisiones, de resolver problemas basados en juicios razonables, debido a que el docente no identifica y selecciona las estrategias didácticas adecuadas para cada momento de la clase, ya sea al inicio, desarrollo o cierre y que desde diferentes puntos de vistas deberán estar canalizadas a establecer iniciativas para reforzar el progreso en su formación académica; se sumó también la escasez del uso de aplicaciones tecnológicas, que intervinieron en el proceso educativo del educando, lo cual ha generó disminución de sus potencialidades.

La educación actual demanda una alta gama de retos en el ámbito formativo, en las instituciones educativas existen muchas falencias en el proceso de enseñanzaaprendizaje, la gran parte de docentes utilizan pocas estrategias en su práctica docente. Por estas razones, se consideró importante un adecuado uso o aplicación de estrategias didácticas aplicadas a las TICs, con la finalidad de mejorar el proceso de aprendizaje, ya que éste le permite el desarrollo de nuevas habilidades y destrezas, despertando en los educandos un total interés por formarse, lo que contribuye a elevar sus niveles de rendimiento académico.

Esta problemática generó incertidumbre en los docentes, generalmente se busca que los educandos alcancen un aprendizaje revelador, se pretende despertar su interés, la motivación y el deseo de aprender. Se enfatizó que estas estrategias son una herramienta indispensable para la educación de calidad, debido a que brindan elementos interactivos y dinámicos para mejorar el proceso de instrucción y de esta forma el estudiante pueda alcanzar un aprendizaje significativo, un aprendizaje para la vida.

Según (Manassero \& Vázquez, 2020, pág. 59) y (Andreu \& García, 2014, pág. 59) se evaluó el pensamiento crítico mediante destrezas, retos, trabajos en grupos, desafiando y motivando al estudiante, y estos instrumentos se consideraron positivos, a través de ello se fundamenta en que se pueden emplear instrumentos efectivos para tomar en cuenta la adquisición del pensamiento crítico de los estudiantes. 
Se precisa que las estrategias didácticas permiten la interacción comunicativa de los estudiantes permitiendo que potencien su pensamiento crítico, conjugando con sus dos componentes principales: el componente cognitivo (habilidades o destrezas) y el componente actitudinal (disposiciones). Esto conlleva a identificar las estrategias más importantes que originen competencias innovadoras y tecnológicas para lograr un cambio radical en la educación, por ello es ineludible recomendar estrategias didácticas con la incorporación de recursos TICs para fomentar el pensamiento crítico en los educandos.

En este artículo se fundamentan las estrategias didácticas, importancia, y tipos, así como también se estudiada el pensamiento crítico, su importancia y el desarrollo del mismo a través de las Tecnología de la información y comunicación más utilizadas en la actualidad.

\section{Estrategias didácticas.}

Según (Lozzada \& Ruiz, 2011, pág. 15), las estrategias didácticas son medios importantes dentro del proceso de enseñanza y aprendizaje, pues orientan de forma exitosa la construcción de los nuevos conocimientos, a la vez, permiten el desarrollo de las competencias en los estudiantes.

Por otra parte, la autora (Mora, 2013, pág. 12) nos muestra que: Las estrategias didácticas, implica la realización de procesos mediante los cuales se eligen, coordinan y aplican las habilidades con el fin que los que reciben conocimiento por medio de ellos para que así puedan adquirir diversas destrezas cognitivas, vinculándose con el aprendizaje significativo.

Las estrategias didácticas implican la elaboración, por parte del docente, de un procedimiento o sistema de aprendizaje cuyas principales características son que se constituya un programa organizado y formalizado y que se encuentre orientado a la consecución de los objetivos específicos previamente establecidos. En su conjunto, demandan establecer una relación dialógica, constante y triangular entre educadores, educandos y metodologías, aunque el educando ignore o no las método y técnicas que utiliza el docente para tal fin, las cuales deben ser conciliadoras y 
reflexivas. Las estrategias didácticas ofrecen al docente grandes posibilidades y expectativas de mejorar la práctica educativa pues tienen una gran importancia en la educación moderna.

\section{Tipos de estrategias didácticas.}

(Cevallos \& Fernández, 2017, pág. 21) Las tendencias enfocadas en el autoaprendizaje se desarrollan por una serie de técnicas llamadas estrategias didácticas que permite hacer distinciones, entre las cuales se puede decir que los tipos de estrategias didácticas son dos una es la participación y la otra es por su alcance, para determinar este concepto.

Las estrategias didácticas representan un apoyo para el desarrollo de los contenidos curriculares, orientan conocimientos previos y promueven nuevos conocimientos. Por la diversidad de estrategias existentes, han sido clasificadas según su utilidad, recordando siempre que las estrategias didácticas son flexibles. Es trascendental destacar el gran impacto que tiene el aplicar estrategias didácticas e interactivas dentro del campo educativo. Entre las principales encontramos:

Tabla 1. Tipos de estrategias didácticas.

\begin{tabular}{|c|c|}
\hline $\begin{array}{l}\text { Aprendizaje } \\
\text { basado en } \\
\text { Problemas }\end{array}$ & $\begin{array}{l}\text { Método de enseñanza-aprendizaje, cuyo punto de partida es un problema } \\
\text { diseñado por el profesor, con la finalidad de que el estudiante lo resuelva } \\
\text { para desarrollar determinadas competencias previamente definidas. }\end{array}$ \\
\hline $\begin{array}{l}\text { Aprendizaje } \\
\text { basado en } \\
\text { Proyectos }\end{array}$ & $\begin{array}{l}\text { ABP es una modalidad de enseñanza que busca evaluar la capacidad de } \\
\text { solucionar un reto por parte de los estudiantes. El profesorado asume } \\
\text { el rol de acompañante o mediador y proporciona apoyo en cuanto al } \\
\text { contenido y al método. }\end{array}$ \\
\hline $\begin{array}{l}\text { Aprendizaje } \\
\text { Colaborativo }\end{array}$ & $\begin{array}{l}\text { Enfoque interactivo de organización del trabajo en el aula, en el cual los } \\
\text { estudiantes son responsables de su aprendizaje y del de sus compañeros, } \\
\text { en una estrategia de corresponsabilidad para alcanzar metas e incentivos } \\
\text { grupales. }\end{array}$ \\
\hline $\begin{array}{l}\text { Flipped } \\
\text { Classroom }\end{array}$ & $\begin{array}{l}\text { El Flipped Classroom (FC) o aula invertida, es un modelo pedagógico que } \\
\text { transfiere el trabajo de determinados procesos de aprendizaje fuera del } \\
\text { aula y utiliza el tiempo de clase, junto con la experiencia del docente, para } \\
\text { facilitar y potenciar otros procesos de adquisición y práctica de } \\
\text { conocimientos dentro del aula. }\end{array}$ \\
\hline
\end{tabular}




\begin{tabular}{|l|l|}
\hline $\begin{array}{l}\text { Aprendizaje } \\
\text { Autónomo }\end{array}$ & $\begin{array}{l}\text { El aprendizaje autónomo refiere al grado de intervención del estudiante en } \\
\text { su propio proceso, estableciendo sus objetivos, procedimientos, recursos, } \\
\text { evaluación y momentos de aprendizaje, desde el rol activo que debe tener } \\
\text { frente a las necesidades actuales de formación. Se refiere a la capacidad } \\
\text { de aprender por uno mismo, sin necesidad de alguien más. }\end{array}$ \\
\hline
\end{tabular}

\section{Importancia de las estrategias didácticas.}

(Pérez, 2015, pág. 13), expresa que: Estas estrategias didácticas tienen como objetivo la composición de la continuación de variadas actividades planificadas y organizadas constantemente permitiendo la construcción de conocimiento escolar. Interviniendo pedagógicamente con mejorar los procesos de aprendizajes y de enseñanzas, con la finalidad de construir el desarrollo de la inteligencia, la efectividad, la conciencia y las competencias.

Las estrategias didácticas tienen una gran importancia en la educación moderna por las posibilidades que ofrecen para definir y organizar el currículo, para presentar los contenidos de las diversas materias, para ser un vehículo de aplicación de las tecnologías de la información y comunicación. Las estrategias didácticas ayudan con la formación continua y diversificada en los educandos. Son la base de una formación

planificada y estandarizada, que permiten a los estudiantes crecer académicamente con criticidad e intrepidez y estimular el aprendizaje porque también despiertan la inquietud por el conocimiento.

\section{Pensamiento crítico.}

Para (Paúl \& Elder, 2018, pág. 36) el pensamiento crítico consiste en reflexionar sobre su pensamiento y al mismo tiempo pensar en cómo mejorarlo. Destacan que naturalmente el hombre no es reflexivo; de aquí que el hombre sea capaz de mejorar la forma en que piensa y pasar de pensador irreflexivo a pensador maestro, el cual se construye desde la aplicación de un sistema que se activa desde el cumplimiento de los estándares intelectuales, los elementos del razonamiento y las características intelectuales.

En la sociedad actual es importante contar con estudiantes críticos capaces de juzgar su contexto y 
proponer soluciones de acuerdo con el mismo, ya que en este mundo tan competitivo la persona que no desarrolle su pensamiento crítico no está a la vanguardia en la transformación educativa. Es por ello que deben desarrollar el pensamiento crítico de una manera única y es allí donde intervienen las estrategias didácticas con un enfoque innovador. Todo estudiante puede tener un punto de vista examinador y reflexivo, así sea algo mínimo, la cual debe ser aprovechado al máximo en su aprendizaje.

\section{Importancia del pensamiento crítico en los estudiantes}

El pensamiento crítico puede llegar a una actuación y a un compromiso propio y social. Por eso su importancia, no sólo en la educación, sino en el mundo profesional. (Jickling, 2002, pág. s/n).

Cuando los maestros reciben una enseñanza actualizada y acorde con los requerimientos de la andragogía moderna, son los primeros elementos multiplicadores para la comunidad a la que sirven. La responsabilidad del personal docente es enorme, se debe planificar, diseñar y ejecutar académicamente un módulo sobre el desarrollo del pensamiento crítico, con esquemas interactivos que permita al docente manejar estas estrategias didácticas que indudablemente motivarán académicamente a los estudiantes. Es porque este pensamiento crítico está ligado a la capacidad de las personas para ser autónomas, con la toma de decisiones. Esto le da importancia al ser el pensamiento crítico la diferencia entre distinguir entre un hecho y una opinión, tomar una decisión acertada o no, entre ser creativos para enfrentar los problemas de cada día, en buscar soluciones coherentes para resolver las dificultades grandes o pequeñas bases para la transformación.

\section{Desarrollo del pensamiento crítico} a través de estrategias didácticas

Para (Aguilar, 2017, pág. 24) "El conocimiento sobre la distribución interhemisférica del procesamiento también supone un avance para el diseño de las estrategias aplicadas a la didáctica".

El aprendizaje significativo, se puede lograr con la aplicación de estrategias de enseñanzas 
recomendadas y seleccionadas por

el docente, en el momento de su participación dentro de las cátedras, ya sea el inicio, desarrollo o cierre de la clase. Dichas estrategias permiten que los estudiantes crezcan académicamente con criticidad y autonomía, estimulan el aprendizaje y despiertan la motivación por el conocimiento y aún más con el acompañamiento de los recursos tecnológicos que facilitan la transmisión de conocimientos alcanzando conocimientos fructíferos. Entre las principales tenemos:

Tabla 2. Recursos tecnológicos que facilitan la transmisión de conocimientos.

\begin{tabular}{|c|c|c|}
\hline Mentimeter & mentimeter & $\begin{array}{l}\text { Aplicación web para interactuar y hacer participar } \\
\text { a una audiencia. }\end{array}$ \\
\hline Mindomo & & $\begin{array}{l}\text { Permite revisar, confeccionar y compartir en línea } \\
\text { una gran diversidad de mapas mentales, } \\
\text { conceptuales y esquemas de manera } \\
\text { colaborativa. }\end{array}$ \\
\hline Padlet & & $\begin{array}{l}\text { Herramienta para crear murales virtuales de forma } \\
\text { colaborativa, en los que se pueden incluir } \\
\text { elementos multimedia, vínculos y documentos. }\end{array}$ \\
\hline Spatial chat & & $\begin{array}{l}\text { Es una plataforma ideal para armar una oficina } \\
\text { virtual, donde se puede acercar e interactuar con } \\
\text { tus compañeros de equipo, armar reuniones o } \\
\text { conferencias. }\end{array}$ \\
\hline Kahoot & & $\begin{array}{l}\text { Servicio web de educación social y gamificada, es } \\
\text { decir, que se comporta como un juego, } \\
\text { recompensando a quienes progresan en las } \\
\text { respuestas con una mayor puntuación que les } \\
\text { catapulta a lo más alto del ranking. }\end{array}$ \\
\hline Educaplay & - educaplay & $\begin{array}{l}\text { Sirve para crear actividades interactivas de una } \\
\text { manera sencilla: crucigramas, sopa de letras, } \\
\text { video quiz, mapa interactivo, test, dictado, } \\
\text { rompecabezas y presentaciones, entre otros. }\end{array}$ \\
\hline Infogram & ar.am & $\begin{array}{l}\text { Permite diseñar y crear infografías para utilizarlas } \\
\text { en nuestros proyectos o presentaciones. }\end{array}$ \\
\hline
\end{tabular}




\begin{tabular}{|c|c|c|}
\hline Moodle & moodle & $\begin{array}{l}\text { Permite a los educadores crear espacios de } \\
\text { enseñanza online y administrar, distribuir y } \\
\text { controlar todas las actividades de formación no } \\
\text { presencial de una entidad educativa. }\end{array}$ \\
\hline Powtoon & sow & $\begin{array}{l}\text { Sirve para la creación de animaciones y todo tipo } \\
\text { de presentaciones en video. }\end{array}$ \\
\hline $\begin{array}{l}\text { Microsoft } \\
\text { Teams }\end{array}$ & & $\begin{array}{l}\text { Plataforma de comunicaciones integradas } \\
\text { ya que a través de esta aplicación de mensajería } \\
\text { se pueden realizar reuniones virtuales, comparti } \\
\text { archivos, realizar llamadas, trabajar en un mismo } \\
\text { documento en tiempo real, chatear y mucho más, }\end{array}$ \\
\hline Zoom & zoon & $\begin{array}{l}\text { Utiliza los servicios de la nube en Internet para } \\
\text { realizar videoconferencias por video, audio o } \\
\text { ambos. }\end{array}$ \\
\hline
\end{tabular}

\section{Métodos}

La investigación tuvo un enfoque cuali-cuantitativa, es decir, que se abordó el tema de estudio desde los aspectos cuantitativos y medibles. Esta investigación recopiló información documental y de campo, puesto que existen datos relevantes recabados en investigaciones previas, y que han sido tomados como base para la misma, reforzándose y orientándose con datos obtenidos en el plano de esta investigación.

Se implementó una investigación exploratoria que estableció tanto las características de orden descriptivo y analítico de las variables del estudio la cual sirvió para estudiar el problema y comprenderlo mejor.

La población de la investigación estuvo conformada por docentes y discentes de Octavo año de Educación General Básica de la Unidad Educativa Fiscal Dr. Francisco Campos Coello.

Para el muestreo de la investigación se consideró a 60 alumnos, 20 docentes y 1 profesional en el área de la Docencia, para determinar la incidencia de las estrategias didácticas en el desarrollo del pensamiento crítico de los estudiantes de Octavo año de EGB

La investigación responde al método Analítico - Deductivo, el cual sirvió para seleccionar el objeto de estudio 
y analizó cada una de sus partes y luego sintetizar nuevamente la información; Inductivo - deductivo este sirvió para construir una investigación sólida y fundamentada correctamente a nivel teórico.

Las técnicas de procesamiento y análisis de los resultados, que se utilizó fue el análisis cuanticualitativo, mediante la recolección de datos a través de cuestionarios de encuestas realizadas en Google Forms con preguntas de selección y una encuesta que se realizó vía Microsoft Teams, para evidenciar los resultados de la investigación.

\section{Resultados y discusión}

En relación con los datos obtenidos de los instrumentos que se aplicaron en esta investigación se presentan los resultados de la encuesta aplicada a docentes solo el $25 \%$ de los docentes encuestados consideran que están capacitados en el uso de estrategias didácticas.

Según (Guapucal \& Jaguandoy, 2015, pág. 27): Dentro de una escuela en constante cambio, los profesores deben potencializar nuevas habilidades, poniendo en práctica nuevas estrategias de enseñanza. En este marco, la formación tanto inicial como permanente debe plantearse cada vez más la necesidad del trabajo común, de reflexionar y crear ante situaciones diversas; pasando de una formación técnica a una formación que posibilite el aprender a aprender.

Los resultados deducen que los docentes no están completamente capacitados en el uso de estrategias didácticas y que sus saberes son superficiales, se denota la demanda de conocimientos y surge la necesidad de prepararse para aumentar el nivel de conocimientos en la aplicación de estrategias como aprendizaje basado en proyectos, aprendizaje basado en problemas, aprendizaje colaborativo, flipped classroom, aprendizaje autónomo entre otros, muchos de los profesores las conocen, pero no las utilizan. De la misma forma, los docentes deben fomentar permanentemente en sus clases, la participación intensa y activa de todos y cada uno de los educandos para que puedan enriquecer y afianzar su nivel de conocimiento. 
Tabla 3. Según su criterio, considera que los docentes están capacitados en el uso de estrategias didácticas.

\begin{tabular}{lcc}
\hline № & Alternativas & Porcentaje \\
\hline 1 & Muy capacitados & $0 \%$ \\
2 & Capacitados & $25 \%$ \\
3 & Algo capacitados & $60 \%$ \\
4 & Poco capacitados & $15 \%$ \\
5 & Nada capacitados & $0 \%$ \\
\hline & Total & $100 \%$ \\
\hline
\end{tabular}

Como se muestran los resultados de la tabla número 2, la mayoría de docentes conocen y utilizan estrategias didácticas, sin embargo, a lo largo de la investigación se puedo denotar que como les falta capacitación, no están aplicando dichas estrategias de manera correcta ni en tiempos precisos de las clases, lo que incide en el proceso de enseñanza - aprendizaje.

(Vaello, 2009, pág. 16) señala que debido a la naturaleza flexible, adaptable y contextualizada de las estrategias didácticas existe la posibilidad de usar una estrategia didáctica en los tres momentos y/o fases de la clase, ya sea en el inicio, desarrollo o cierre. La selección de las estrategias didácticas tiene sus fundamentos en los constructos teóricos de (Díaz \& Hernandez, 1999).
Uno de los mayores desafíos para los docentes en su práctica pedagógica, es la implementación de estrategias didácticas, que les permita desarrollar de mejor manera su proceso de enseñanzaaprendizaje, permitiéndoles seleccionar las técnicas y recursos tecnológicos acordes a la estrategia didáctica innovadora a utilizar; es necesario que los docentes conozcan e implementen estrategias didácticas adecuadas y en el momento oportuno en sus clases, para que los estudiantes desarrollen su conocimiento, creatividad y estimulen el pensamiento creativo y dinámico. 
Tabla 4. De las siguientes estrategias didácticas. ¿Cuáles conoce y utiliza?

\begin{tabular}{|c|c|c|c|c|c|c|c|}
\hline \multirow[t]{2}{*}{ № } & \multirow[t]{2}{*}{ Alternativas } & $\begin{array}{l}\text { Aprendizaje } \\
\text { Basado en } \\
\text { Problemas }\end{array}$ & $\begin{array}{l}\text { Aprendizaje } \\
\text { Basado en } \\
\text { Proyectos }\end{array}$ & $\begin{array}{c}\text { Aprendizaje } \\
\text { Autónomo }\end{array}$ & $\begin{array}{c}\text { Flipped } \\
\text { Classroom }\end{array}$ & $\begin{array}{c}\text { Trabajo } \\
\text { Colaborativo }\end{array}$ & Otras \\
\hline & & Porcentaje & Porcentaje & Porcentaje & Porcentaje & Porcentaje & Porcentaje \\
\hline 1 & $\begin{array}{l}\text { Conocen y } \\
\text { utilizan }\end{array}$ & $50 \%$ & $90 \%$ & $55 \%$ & $80 \%$ & $90 \%$ & $70 \%$ \\
\hline 2 & $\begin{array}{l}\text { Conocen, } \\
\text { pero no } \\
\text { utilizan } \\
\text { Desconocen }\end{array}$ & $25 \%$ & $10 \%$ & $45 \%$ & $20 \%$ & $0 \%$ & $25 \%$ \\
\hline 3 & $\begin{array}{l}\text { y desean } \\
\text { utilizar } \\
\text { No conocen }\end{array}$ & $25 \%$ & $0 \%$ & $0 \%$ & $0 \%$ & $10 \%$ & $0 \%$ \\
\hline 4 & $\begin{array}{l}\text { y no desean } \\
\text { utilizar }\end{array}$ & $0 \%$ & $0 \%$ & $0 \%$ & $0 \%$ & $0 \%$ & $5 \%$ \\
\hline & Total & $100 \%$ & $100 \%$ & $100 \%$ & $100 \%$ & $100 \%$ & $100 \%$ \\
\hline
\end{tabular}

Los estudiantes encuestados revelaron con un 22\%, que de manera muy frecuente los docentes motivan la participación en sus clases planteando problema en donde ellos deben dar soluciones y opiniones.

Según (Lucio, 2015, pág. 9) las estrategias de aprendizaje son procedimientos (conjuntos de pasos, operaciones o habilidades) que un aprendiz emplea en forma consciente, controlada e intencional como instrumento flexible para aprender significativamente y solucionar problemas."
La resolución de problemas resulta ser una de las problemáticas que en estos últimos tiempos está siendo abordada con gran interés y preocupación por la comunidad educativa. Los docentes tienen una ardua labor al utilizar la resolución de problemas como estrategia de enseñanza didáctica, las mismas que invita al estudiante a razonar, crear, reflexionar, analizar y razonar, para concebir una técnica en base a una estrategia didáctica que le permita obtener la solución de los problemas planteados, potenciar habilidades y destrezas claves para la vida adulta como la toma de decisiones y una mejor capacidad de investigación y de comunicación. 
Tabla 5. ¿Con qué frecuencia los docentes plantean problemas en donde el estudiante propone soluciones?

\begin{tabular}{lcc}
\hline № & Alternativas & Porcentaje \\
\hline 1 & Muy frecuentemente & $22 \%$ \\
2 & Frecuentemente & $40 \%$ \\
3 & Ocasionalmente & $18 \%$ \\
4 & Raramente & $15 \%$ \\
5 & Nunca & $5 \%$ \\
\hline & Total & $100 \%$ \\
\hline
\end{tabular}

Los estudiantes interrogados revelaron con un $27 \%$ que, el profesor siempre realiza actividades grupales en clases.

Es fundamental, para ejercitar el pensamiento crítico, la interacción con los que rodean al individuo y, por tanto, el trabajo colaborativo debe ser una parte integrante de este proceso. El diálogo o debate fomenta tanto el intercambio de distintos puntos de vista como la necesidad de estructurar la argumentación para realizar el intercambio de dichos puntos de vista (Beyer, 1990, pág. 15)

Resulta ineludible que el docente realice actividades grupales que fomenten la cohesión de los estudiantes que participan en cada uno de los temas planteados, logrando de manera mancomunada que cada uno de ellos sumen sus esfuerzos para alcanzar un mismo objetivo, permitiendo fomentar la interacción, acelerar el aprendizaje, mejorar las destrezas sociales y solucionar problemas de manera rápida y eficiente, en el ámbito de la educación se han convertido en una alternativa metodológica de gran éxito. En este sentido, a través del desarrollo de dinámicas de grupo en el aula se pretende que los estudiantes puedan asimilar los contenidos trabajados en las sesiones teóricas de un modo práctico, siendo su objetivo esencial es que el estudiante aprenda y se divierta al mismo tiempo. 
Tabla 6. ¿Con qué frecuencia el profesor realiza actividades en grupo?

\begin{tabular}{lcc}
\hline $\mathbf{N}$ No & Alternativas & Porcentaje \\
\hline 1 & Siempre & $27 \%$ \\
2 & Casi siempre & $20 \%$ \\
3 & Ocasionalmente & $20 \%$ \\
4 & Pocas veces & $22 \%$ \\
5 & Nunca & $12 \%$ \\
\hline & Total & $100 \%$ \\
\hline
\end{tabular}

De la entrevista realizada a la profesional en el área nos mencionó que en las TICs no deberían estar aisladas de las estrategias didácticas, sino más bien deberían ser parte fundamental, desencadenando las diferentes estrategias como el trabajo colaborativo, el trabajo en red las cuales deben ir trabajadas mancomunadamente, entre ellas el Mentimeter, Padlet, Powton, Quizziz, Kahoot, Prezzi, Google Drive, Microsoft Teams, Infografías, etc.

De igual manera, (Monsalve y Monsalve, 2015, pág. 27) consideran que: "Para integrar las TIC en la enseñanza se requiere de una serie de condiciones, como la formación del profesor en su uso y posibilidades para innovar y la actitud positiva hacia los procesos generados de su implementación", lo cual es totalmente consistente con la posición de (Santiago et al., 2013, pág. 27) quienes consideran que para incorporar las TIC a los procesos de aprendizaje se requiere disposición e interés en el tema, ya que muchas veces se presentan actitudes de resistencia que no permiten un uso e incorporación de dichas herramientas en el aula de clase.

Las TICs son la innovación educativa en la actualidad, permiten a los docentes y alumnos cambios determinantes en el quehacer diario del aula y en el proceso de enseñanza-aprendizaje, pues son herramientas necesarias y valiosas, facilitan la integración de espacios virtuales adaptándose a las capacidades del alumno, a su ritmo de aprendizaje y a sus conocimientos previos mediante herramientas digitales como 
Microsoft Teams, Padlet, Microsoft Office 365, Mentimeter, Genially, Canvas, etc. El uso de las TICs en el escenario educativo actual y las estrategias didácticas basadas en ellas, hacen que las clases se conviertan en un ejercicio de la democracia interactiva, promoviendo una actitud activa y participativa, las mismas que permiten entrar a un mundo nuevo lleno de información de fácil acceso para los docentes y discentes.

\section{Conclusiones}

Al evaluar el conocimiento de las estrategias didácticas que poseen los docentes, se identifica que sus dominios conceptuales $y$ metodológicos no son integrales. Es de vital importancia la implementación de una gran variedad de estrategias didácticas en clases, tales como: el aprendizaje basado en proyectos, aprendizaje basado en problemas, aprendizaje colaborativo, flipped classroom, aprendizaje autónomo, etc. las cuales ayudan a activar los conocimientos previos en los estudiantes, y crean ambientes de aprendizaje enriquecedores, propicios para aprender; en donde los estudiantes se sientan motivados, entusiasmados y comprometidos, con participación activa en todos los aspectos, siendo analíticos, críticos y reflexivos.

Se ha demostrado que el desarrollo del pensamiento crítico se genera a partir de la aplicación aprendizaje basado en proyectos, aprendizaje basado en problemas, aprendizaje colaborativo, flipped classroom, aprendizaje autónomo, creado como actividad inherente a las estrategias didácticas; por tanto, se valora la relación armoniosa y efectiva para fomentar habilidades del pensamiento crítico, superando de manera progresiva las prácticas docentes tradicionales, aportando a la formación de estudiantes críticos, reflexivos, y autónomos que disfrutan del aprendizaje. Es importante destacar que el uso y aplicación de estrategias didácticas afianza el nivel de pensamiento crítico y reflexivo de los educandos. Las destrezas del pensamiento crítico son claves para aprender sobre cualquier área.

Es necesario enfatizar que las estrategias didácticas con la incorporación de recursos TICs como: Mentimeter, Mindomo, 
Genially, Canvas, Padlet, Spatial chat, Kahoot, Educaplay, Infogram, Moodle, Powtoon, Socrative, Microsoft Teams, Zoom, entre otras; ayudan a desarrollar el pensamiento crítico de los estudiantes y no solo permiten que se apropien de los conocimientos, sino que desarrollen habilidades, formen valores $y$ adquieran estrategias que les permitan actuar de forma independiente, comprometida y creadora, para resolver los problemas a los que deberá enfrentarse en su futuro personal y profesional. Las estrategias didácticas innovadoras acompañadas de las TICs son el vehículo del aprendizaje tanto para docentes como para los estudiantes quienes son nativos digitales.

\section{Bibliografía}

Aguilar. (2017). Estrategias didácticas en el aprendizaje significativo del subnivel medio. Guayaquil: Universidad de Guayaquil.

Andreu, \& García. (2014). Metodologías activas para el desarrollo del pensamiento crítico en los estudiantes de la básica media de la escuela de educación general básica Charapotó $n^{\circ} 75$. Portoviejo:
Universidad San Gregorio de Portoviejo.

Beyer. (1990). Diseño de una propuesta didáctica para desarrollar el pensamiento crítico y creativo, utilizando un sistema no graduado, en la Educación Secundaria. Sabadell: Universidad Internacional de la Rioja.

Cevallos, \& Fernández. (2017). Estrategias didácticas en el aprendizaje significativo del subnivel medio. Guayaquil: Universidad de Guayaquil.

Chin, V. (2013). Aplicación de la estrategia didáctica "estudio de casos" para un curso de biología general en la Universidad Nacional de Colombia. Medellín: Universidad Nacional de Colombia.

Díaz Barriga, F. y Hernández Rojas, G. (2010). Estrategias Docentes para un aprendizaje significativo. 3a. Edición. México: Mac Graw Hill.

Guapucal, \& Jaguandoy. (2015). Estrategias de enseñanza del docente en las áreas básicas: una mirada al aprendizaje escolar. Eleuthera, 27.

Jickling, W. \&. (2002). El Pensamiento Crítico desde la Perspectiva de los Docentes Universitarios. Obtenido de https://scielo.conicyt.cl/scielo. 
php?script=sci_arttext\&pid=S

0718-07052018000100089

Lozzada, \& Ruiz. (2011). Facultad de ciencias de la educación y humanidades. Lima.

Lucio. (2015). Estrategias que permitan mejorar la participación activa durante el proceso de aprendizaje en estudiantes de Formación Docente. Estelí: Universidad Nacional Autónoma de Nicaragua.

Manassero, \& Vázquez. (2020). Metodologías activas para el desarrollo del pensamiento crítico en los estudiantes de la básica media de la escuela de educación general básica Charapotó $n^{\circ} 75$. Portoviejo: Universidad San Gregorio de Portoviejo.

Monereo. (1999). Estrategias didácticas para promover el aprendizaje activo en los estudiantes de noveno" D". Babahoyo: Universidad Técnica de Babahoyo.

Mora. (2013). Estrategias didácticas en el proceso de enseñanza y aprendizaje. Guayaquil: Universidad de Guayaquil.

Paúl, \& Elder. (2018). Relación entre habilidades de pensamiento. Revista Latinoamericana de Educación, 36.
Pérez, J. (2014). Ventajas Desventajas Estrategias Didacticas. Scrib.

Pérez. (2015). Universidad de Guayaquil. Guayaquil.

Richard, Paul y Elder, Linda. (2003). Bolsilibro para estudiantes sobre cómo estudiar y aprender Fundación para el Pensamiento Crítico. Disponible en internet en: www.criticalthinking.org

Romero, G. (2009). "La utilización de estrategias didácticas en clase". Revista Digital Innovación y Experiencias Educativas, No. 23, pp. 1-8

Roque. (2016). Las estrategias didácticas y su influencia en el aprendizaje significativo de los estudiantes de Quinto Año de Educación General Básica. Guayaquil: Universidad Laica Vicente Rocafuerte.

Sequeira, A. \&. (2016). Estrategias metodológicas que facilitan el proceso de enseñanza aprendizaje. Obtenido de http://repositorio.unan.edu.ni/ 1638/1/10564.pdf Pág. 34. 